\title{
The Role of Presynaptic Dynamics in Processing of Natural Spike Trains in Hippocampal Synapses
}

\author{
Umasankar Kandaswamy, ${ }^{1}$ Pan-Yue Deng, ${ }^{1}$ Charles F. Stevens, ${ }^{2}$ and Vitaly A. Klyachko ${ }^{1}$ \\ ${ }^{1}$ Departments of Biomedical Engineering, and Cell Biology and Physiology, Washington University School of Medicine, St. Louis, Missouri 63110, and ${ }^{2}$ Salk \\ Institute for Biological Studies, La Jolla, California 92037
}

\begin{abstract}
Short-term plasticity (STP) represents a key neuronal mechanism of information processing. In excitatory hippocampal synapses, STP serves as a high-pass filter optimized for the transmission of information-carrying place-field discharges. This STP filter enables synapses to perform a highly nonlinear, switch-like operation permitting the passage and amplification of signals with place-field-like characteristics. Because of the complexity of interactions among STP processes, the synaptic mechanisms underlying this filtering paradigm remain poorly understood. Here, we describe a simple mechanistic model of STP, derived in large part from basic principles of synaptic function, that reproduces this highly nonlinear synaptic behavior. The model, formulated in terms of release probability, considers the interactions between calcium-dependent forms of presynaptic enhancement and their impact on vesicle pool dynamics, which is described using a two-pool model of vesicle recruitment. By considering the interdependency between release probability and various forms of STP, the model attempts to provide a realistic coupling among major presynaptic processes. The model parameters are first determined using synaptic dynamics during constant-frequency stimulation. The model then accurately reproduces all major characteristics of the synaptic filtering paradigm during natural stimulus patterns without free parameters. An elimination approach is then used to identify the contribution of each STP component to synaptic dynamics. Based on this analysis, the model predicts strong calcium dependence of synaptic filtering properties, which is verified experimentally in rat hippocampal slices. This simple model may thus offer a useful framework to further investigate the role of STP in neural computations.
\end{abstract}

\section{Introduction}

Short-term plasticity (STP) comprises several rapid synaptic processes that operate on millisecond-to-minute timescales and modulate synaptic strength in an activity-dependent manner (Zucker and Regehr, 2002). This rapid, bidirectional, and reversible modulation of synaptic properties is believed to function as one of the fundamental neuronal mechanisms of information processing in the brain. The specific computations that STP performs during neural activity have been mostly studied in systems in which a single form of STP dominates synaptic dynamics, including input compression and gain control in cortical neurons (Abbott et al., 1997), sound localization in the auditory brainstem (Cook et al., 2003), and sensory adaptation in primary visual (Chance et al., 1998) and somatosensory cortices (Chung et al., 2002). However, the role of STP in information processing has been more difficult to establish in central synapses expressing multiple interacting forms of STP. The contributions of specific STP components to synaptic dynamics thus remain incompletely understood.

Received Aug. 3, 2010; revised Sept. 16, 2010; accepted Sept. 22, 2010.

This work was supported in part by a Whitehall Foundation grant to V.K. We thank Drs. S. Mennerick, B. Raman, V.Cavalli, and D. Owyoung for helpful comments on the manuscript.C.S.,U.K., and V.K. designed the model, V.K. and P.D. carried out the experiments, U.K. and V.K. performed computations, and V.K. wrote the article.

Correspondence should be addressed to Vitaly A. Klyachko, Department of Biomedical Engineering, Department of Cell Biology and Physiology, Washington University School of Medicine, St. Louis, M0 63110. E-mail: klyachko@wustl.edu.

DOI:10.1523/JNEUROSCI.4050-10.2010

Copyright $\odot 2010$ the authors $\quad 0270-6474 / 10 / 3015904-11 \$ 15.00 / 0$
The relatively well understood information encoding in the hippocampus makes the excitatory hippocampal synapses an attractive platform to elucidate the contributions of STP processes to synaptic computations. The activity of many pyramidal cells in the CA3-CA1 areas of the hippocampus encodes information about the animal's location via high-frequency spike discharges at certain places in the environment, known as place-fields (O'Keefe and Dostrovsky, 1971). Using such natural spike patterns recorded in active rodents, we have shown that STP in hippocampal synapses acts as a high-pass filter optimized for transmission of information-carrying place-field discharges (Klyachko and Stevens, 2006a). This highly nonlinear switch-like filtering paradigm is characterized by a rapid increase and saturation of synaptic strength during high-frequency input. Saturation of synaptic strength occurs at frequencies above $\sim 7 \mathrm{~Hz}$, leading to largely content-independent processing of place-field discharges, which typically have higher spike frequencies. Because of the complex interdependencies among the STP components that determine synaptic dynamics in this system, the underlying synaptic mechanisms of this filtering paradigm remain poorly understood, preventing incorporation of these dynamic processes into larger-scale models of neural networks.

We therefore sought to account for the reciprocal interdependencies among STP components and synaptic release probability with a simple, yet mechanistically relevant model of STP. This model builds on and extends several previous STP models (Magleby and Zengel, 1982; Zengel and Magleby, 1982; Bertram et al., 1996; Tsodyks and Markram, 1997; Varela et al., 1997; Weis 
et al., 1999; Dittman et al., 2000; Hempel et al., 2000; Pan and Zucker, 2009). We show that once tuned, the model correctly predicts synaptic dynamics during natural stimulus trains without free parameters. The model correctly reproduces all main characteristics of the nonlinear synaptic filtering pattern experimentally observed. Elimination of individual forms of STP from the model reveals the contributions of each STP process to synaptic dynamics. The model further predicts strong calcium dependence of the synaptic filtering pattern that we verify experimentally in hippocampal slices. This simple model thus captures many aspects of rapid synaptic dynamics in hippocampal synapses and may offer a useful framework to further investigate the role of STP in synaptic computations.

\section{Materials and Methods}

Preparation. Transverse hippocampal slices $(400 \mu \mathrm{m})$ were prepared as previously described (Pouille and Scanziani, 2001; Losonczy et al., 2002). In brief, slices were cut on a Leica Vibratome in ice-cold artificial CSF, incubated for $\sim 1 \mathrm{~h}$ at $32^{\circ} \mathrm{C}$, and then kept at the room temperature for $1-4 \mathrm{~h}$ before recordings. The CA3 area was surgically separated in each slice with an incision to prevent recurrent excitation. During the experiments, the bath temperature was continuously monitored and adjusted to $33-34^{\circ} \mathrm{C}$ with an automatic in-line heater controller with a rapid feedback.

Whole-cell recordings. Synaptic currents were recorded in whole-cell configuration from CAl pyramidal cells in rat hippocampal slices at $33-34^{\circ} \mathrm{C}$ using an Axopatch $200 \mathrm{~B}$ amplifier. Synaptic activity was induced by stimulation of Schaffer collaterals via a bipolar electrode placed in the stratum radiatum, and whole-cell currents were recorded at the holding potential of $-70 \mathrm{mV}$. Micropipettes $(3-6 \mathrm{M} \Omega$ ) were filled with solution containing the following (in $\mathrm{mM}$ ): $130 \mathrm{~K}$-Gluconate, $5 \mathrm{KCl}, 10$ $\mathrm{NaCl}, 10$ HEPES, 5 EGTA, 2.5 MgATP, 0.3 LiGTP, pH 7.25 (290-300 mOsm). Access resistance was continuously monitored, and cells with unstable access resistance ( $>20 \%$ change) were excluded from analysis. All recordings were made in the presence of NMDA receptor antagonist AP-5 $(50 \mu \mathrm{M})$ to prevent possible long-term effects and either gabazine (2 $\mu \mathrm{M})$ or bicuculine $(20 \mu \mathrm{M})$ to block inhibitory transmission.

Data analysis and presentation. Stimulation patterns were presented multiple times to the same cell, separated by $2 \mathrm{~min}$ control sections at 0.1-0.2 Hz. Each subset of data was normalized to an average of five control EPSCs immediately preceding each train. Data were analyzed using custom software locally written in Matlab. To account for the overlap of postsynaptic currents that occurs at short interspike intervals, a template of EPSC waveform was first created for each train presentation by averaging responses separated by at least $100 \mathrm{~ms}$ from their neighbors and normalized to their peak values. Every EPSC in the train then was approximated by a template waveform scaled to the peak of the current EPSC and its contribution to synaptic response was subtracted. For the model fitting, experimentally measured synaptic responses to constantfrequency trains were smoothened using a second-order polynomialbased approximation in Matlab to remove the effects of noise. All data are presented as mean \pm SEM. Statistical significance was evaluated using the two-tailed $t$ test.

Natural spike patterns. The natural stimulation patterns used here represent timings of action potential firing recorded in vivo from the hippocampal place cells of awake, freely moving rats (generously provided by Drs. Fenton and Muller) (Fenton and Muller, 1998). Natural stimulation patterns were presented multiple times to the same cell, separated by 2 min control sections at $0.1-0.2 \mathrm{~Hz}$. Spikes with the interspike intervals (ISIs) $<10 \mathrm{~ms}$ were treated as a single stimulus, because the delay between the action potential firing and the peak of postsynaptic currents/ potentials prevented resolution of individual synaptic responses at shorter ISIs. Such treatment does not significantly affect synaptic responses to natural stimulus trains as we previously have shown (Klyachko and Stevens, 2006a).
Model of short-term plasticity

Release probability. To establish mechanistically relevant interdependencies of presynaptic STP processes, we start by considering the dependence of release probability of a synapse $P_{\text {syn }}$ on the fusion probability $\pi$ of individual vesicles and on the number of vesicles in the readily releasable pool $\left(n_{\mathrm{RRP}}\right)$. In the initial model formulation, we will assume monovesicular release for simplicity and then extend and test the model for the case of multivesicular release (below). If average vesicle fusion probability is $\pi$, then the probability that a vesicle is not released is $(1-\pi)$, and the probability that none of the $n_{\mathrm{RRP}}$ vesicles is released is, assuming independence $(1-\pi)^{n_{\mathrm{RRP}}}$. Thus, in the mono-vesicular release framework, the overall release probability of a synapse is given by the following:

$$
P_{\text {syn }}=1-(1-\pi)^{n_{\mathrm{RRP}}} .
$$

The above gives the probability that at least one vesicle will be released, but this is identical to the release probability if at most one vesicle can be released.

Experimentally observed changes in synaptic strength, $S_{\text {syn }}$, during stimulus trains are defined as relative changes in the EPSCs normalized to the average control EPSC recorded at low-frequency stimulation, so that synaptic strength $S_{\text {syn }}$ is related to the release probability $P_{\text {syn }}$ following the $i$ th stimulus as follows:

$$
S_{\mathrm{syn}}(i)=\frac{\operatorname{EPSC}(i)}{\operatorname{EPSC}(0)} \approx \frac{N P_{\mathrm{syn}}(i) q}{N P_{\mathrm{syn}}(0) q}=\frac{P_{\mathrm{syn}}(i)}{P_{\mathrm{syn}}(0)}
$$

assuming that quantal size $q$ and the number of release sites $N$ remains constant within the short timescale relevant to STP (but see Multivesicular release below). The experimentally measured EPSCs represent the average synaptic response of a population of synapses, and this approach ignores the stochastic nature of release and the population distribution of synaptic parameters. Similar to the previous models (Magleby and Zengel, 1982; Zengel and Magleby, 1982; Tsodyks and Markram, 1997; Varela et al., 1997; Markram et al., 1998; Dittman et al., 2000; Pan and Zucker, 2009), we will thus consider all synaptic parameters, including $P_{\text {syn }}$, as the deterministic average of a population of similar synapses. This situation may be relevant, for example, during simultaneous activation of a synaptic population by an ensemble firing of CA3 pyramidal cells, which is typical in the hippocampal circuit (Wilson and McNaughton, 1993; Guzowski et al., 2004).

Contributions of STP components to vesicle fusion probability. The two main components of short-term synaptic enhancement, facilitation $\Phi$ and augmentation $A$, are believed to act by increasing the release probability for synaptic vesicles (Zucker and Regehr, 2002). To derive the form of contribution of $\Phi$ and $A$ to vesicular release probability $\pi$, we considered the vesicle fusion produced by an increase in intraterminal calcium following synaptic activation in terms of rate theory. The probability $\pi$ for vesicle fusion is given by a fusion rate $\rho$ times the duration of calcium action $\tau_{\mathrm{Ca}}$ according to:

$$
\pi=\rho \tau_{\mathrm{Ca}}=\gamma e^{-\frac{U_{C a}}{k T}}
$$

where $U_{C a}$ is the energy barrier that must be overcome for a fusion event when calcium is present, $T$ is the absolute temperature, $k$ is Boltzmann's constant, and $\gamma$ is proportional to the resting release rate. Under resting conditions in the absence of activity, the energy barrier $U_{C a}$ is very high (very low resting fusion rate) but is lowered as a result of calcium influx during the action potential due to the calcium binding to a calcium sensor for release, believed to be synaptotagmin I (Neher and Sakaba, 2008). When facilitation is present, the energy barrier is further lowered so that the barrier height is $U_{C a}+U_{\Phi}$, where $U_{\Phi}$ is the contribution to the barrier for vesicle fusion from the second calcium sensor known to be separate from synaptotagmin I (Geppert et al., 1994; Goda and Stevens, 1994). With facilitation present, then, the vesicle fusion probability is

$$
\pi \sim e^{-\frac{U_{C a}+U_{\Phi}}{k T}}=e^{-\frac{U_{C a}}{k T}} e_{\sim \lambda}^{e^{-\frac{U_{\Phi}}{k T}}=\lambda \Phi}=\lambda \Phi
$$


where $\lambda$ is the basal vesicle fusion probability without facilitation present and $\Phi$ is the contribution from facilitation. Thus, by analogy to the operation of synaptotagmin $I$, the contribution by facilitation to vesicular release probability is multiplicative. Assuming that facilitation and augmentation are mediated by different calcium-binding molecules (Rosenmund et al., 2002; Sippy et al., 2003; Junge et al., 2004), augmentation acts independently to lower the energy barrier for vesicle fusion and thus also multiplies the basal fusion probability $\lambda$. Then the fusion probability $\pi$ in the presence of both facilitation and augmentation is as follows:

$$
\pi=\lambda \Phi \mathrm{A} .
$$

Facilitation. Our treatment of facilitation $\Phi$ closely follows formulations proposed in previous models (Mallart and Martin, 1967; Bertram et al., 1996; Wang, 1999; Dittman et al., 2000; Hempel et al., 2000) and is consistent with an assumption that facilitation is associated with a calcium-binding molecule that translates elevations of presynaptic residual calcium levels into effective increase in release probability. The state of facilitation is specified by the facilitation variable $\varphi$ that may represent the amount of a calcium-bound facilitation molecule. $\varphi$ is assumed to increase instantaneously by amount $h_{f}$ with each impulse and then exponentially decay with a time constant $\tau_{f}$. The value of facilitation variable $\varphi$ can be calculated sequentially for each consecutive spike in a sequence as follows:

$$
\varphi_{k+1}=h_{f}+\varphi_{k} e^{-\Delta t / \tau_{f}},
$$

where $\Delta t$ is the interstimulus interval between $k+1$ and $k$ th stimuli. The decay of the facilitation variable as a function of time $t$ when there is no stimulation is as follows:

$$
\varphi(t)=\varphi(0) e^{-t / \tau_{f}}
$$

This description of facilitation variable $\varphi$ is based on experimental evidence for an exponential decay of facilitation measured with a pairedpulse protocol (Zucker and Regehr, 2002). We further assumed a DodgeRahamimoff-type relationship that couples the state of occupancy of the facilitation molecule $\varphi$ and effective facilitation $\Phi$ :

$$
\Phi(\varphi)=\left(\frac{\varphi}{1+\eta \varphi}\right)^{\theta}+1,
$$

where $\theta$ is the degree of cooperativity and the constant $\eta$ determines the value of the effective facilitation $\Phi$ for large values of $\varphi$. The high-affinity calcium sensor presumably responsible for facilitation has been shown, like the low-affinity sensor that mediates usual release, to be well described with $\theta=4$ (Goda and Stevens, 1994). Similarly to the previous model (Dittman et al., 2000), however, we found no significant improvement in the model performance for the power law relationship in Equation 8 versus the linear relationship and thus kept $\theta=1$ for simplicity. Note that in this formulation, the basal value of $\Phi$ in the absence of activity is set to 1 .

Our initial experiments showed that adequate model performance required the presence of a second, very rapid component of facilitation (supplemental Fig. 1, available at www.jneurosci.org as supplemental material). We therefore added a second component of facilitation designated $\Phi_{2}$ that had the same formulation as described above except for a different time constant $\tau_{\mathrm{f} 2}$ and amount of increase $h_{\mathrm{f} 2}$ per stimulus. Assuming independent action of facilitation components $\Phi_{1}$ and $\Phi_{2}$ on vesicle fusion probability, the total facilitation is then given by the following:

$$
\Phi=\Phi_{1} \Phi_{2}
$$

Augmentation. The slower calcium-dependent component of synaptic enhancement, known as augmentation, has been previously incorporated in phenomenological models of STP in the neuromuscular junction (NMJ) (Magleby and Zengel, 1982; Zengel and Magleby, 1982; Kalkstein and Magleby, 2004) but has not been widely considered in more detailed models of STP at central synapses. Here, augmentation is assumed to behave much like facilitation, being associated with some calcium-binding molecule that translates elevation in residual calcium levels into effective augmentation. As with $\varphi$, the augmentation variable $\alpha$ can be calculated sequentially for each consecutive spike as follows:

$$
\alpha_{k+1}=h_{\alpha}+\alpha_{k} e^{-\Delta t / \tau_{\alpha}},
$$

where $h_{\alpha}$ is the increase in the amount of calcium-bound augmentation molecule with each impulse, and $\tau_{\alpha}$ is the decay constant of augmentation variable determined by the decay of intraterminal calcium. In the absence of stimulation, the augmentation variable decays exponentially according to the following:

$$
\alpha(t)=\alpha(0) e^{-t / \tau_{\alpha}} .
$$

The state of augmentation variable results in effective augmentation $A$ as given by the following:

$$
A(\alpha)=\left(\frac{\alpha}{1+\mu \alpha}\right)^{\theta}+1,
$$

where the constant $\mu$ determines the maximum amount of effective augmentation A. Similarly to treatment of facilitation, we kept $\theta=1$ for simplicity, and the basal value of $\mathrm{A}$ in the absence of activity was also set to 1 .

Short-term depression. Several presynaptic and postsynaptic mechanisms have been proposed to contribute to short-term synaptic depression in different systems (Zucker and Regehr, 2002). The relative contributions of different mechanisms vary widely in different synapses; in excitatory hippocampal synapses, vesicle depletion is believed to be the dominant contributor to short-term synaptic depression during trains of activity (Dobrunz and Stevens, 1997; Stevens and Wesseling, 1998; Sara et al., 2002). Here, we consider a sequential two-pool scheme for vesicle refilling consisting of a RRP with a size $n_{\text {RRP }}$ that refills itself with a time constant $\tau_{d 1}$ and is also refilled from a larger "recycling" pool of $n_{\mathrm{REC}}$ vesicles with a time constant of $\tau_{d 2}$. The recycling pool in turn is refilled from a much larger reserve pool. In our treatment of depletion, we assume that the size of the RRP is decreased on average by an amount equal to $P_{\text {syn }}$ with each impulse in a single-vesicle release framework. Let us consider first the RRP itself without contributions from the second pool; when the RRP size $n_{\mathrm{RRP}}$ has been reduced during a train of stimuli to a value $n_{\mathrm{RRP}}(i)$ after $i$-th stimulus in a train, it recovers toward the full RRP pool $n_{\mathrm{RRP}}(0)$ according to:

$$
n_{\mathrm{RRP}}(i+1)=n_{\mathrm{RRP}}(0)-\left(n_{\mathrm{RRP}}(0)-n_{\mathrm{RRP}}(i)\right) e^{-t / \tau_{D 1}} .
$$

Taking into account the additional refilling of the RRP from the second pool $\left(n_{\text {REC }}\right)$ and the average amount of vesicle use per stimulus (given by $\left.P_{\text {syn }}\right)$, the state of the RRP can be calculated sequentially for each consecutive stimulus as follows:

$n_{\mathrm{RRP}}(i+1)=n_{\mathrm{RRP}}(0)-\left(n_{\mathrm{RRP}}(0)-n_{\mathrm{RRP}}(i)\right) e^{-\Delta t / \tau_{D 1}}+\xi n_{\mathrm{REC}}(i+1) e^{-\Delta t / \tau_{D 2}}-P_{s y n}$,

where $\xi=\frac{n_{\mathrm{RRP}}(0)}{n_{\mathrm{REC}}(0)}\left(1-e^{-\left(n_{\mathrm{RRP}}(0)-n_{\mathrm{RRP}}(\mathrm{i})\right)}\right)$ is a switch function based on the refilling state of the RRP introduced to prevent the overfilling of the RRP from the recycling pool during periods of low activity.

The second pool depletes by transferring vesicles to the RRP and at the same time slowly replenishes from another large reserve vesicle pool. To simplify formulation, we described the state of the recycling pool by a shrinkage rate representing the difference between its depletion and replenishment as follows:

$$
n_{\mathrm{REC}}(i+1)=n_{\mathrm{REC}}(i) e^{-\Delta t / \tau_{D 3}},
$$

where $\tau_{D 3}$ is the rate at which the second pool shrinks.

Multivesicular release. Recent studies have provided evidence for the presence of multivesicular release (MVR) in several synaptic preparations (Tong and Jahr, 1994; Auger et al., 1998; Oertner et al., 2002; Singer et al., 2004; Foster et al., 2005; Christie and Jahr, 2006) and suggest that the prevalence of MVR may depend on the release probability of the 
synapse. Because the relationship between the release probability and the extent of MVR in hippocampal synapses is not known, we considered two simplified descriptions of MVR based on the assumptions that MVR is due to simultaneous release from multiple release cites, and the number of simultaneously active release sites is either constant (follows a uniform distribution) or follows a Poisson distribution.

Our initial formulation then can easily be generalized for the case of MVR, in which case $P_{\text {syn }}$ represents a release probability for a single release site $P_{\mathrm{rs}}$ and is multiplied by the number of active release sites:

$$
\begin{gathered}
P_{\text {syn }}=P_{\mathrm{rs}} \\
S_{\mathrm{syn}}(i)=\frac{\operatorname{EPSC}(i)}{\operatorname{EPSC}(0)} \approx \frac{N(i) P_{\mathrm{rs}}(i) q}{N(0) P_{\mathrm{rs}}(0) q}=k \frac{P_{\mathrm{rs}}(i)}{P_{\mathrm{rs}}(0)},
\end{gathered}
$$

where $k$ represents the change in the number of simultaneously active release sites. Assuming that the release sites share the same vesicle population within the synapse, the amount of vesicle use per stimulus is then determined by $w P_{\text {syn }}$ in Equation 14, where $w=1,2,3 \ldots$ and is the number of simultaneously active release sites. Our analysis showed that the model fitted constant-frequency synaptic responses equally well in assumptions of mono-vesicular release or MVR in the two formulations we considered (for two examples, see supplemental Fig. $2 A, B$, available at www.jneurosci.org as supplemental material). Our model thus neither excludes nor proves the possibility that MVR significantly contributes to rapid synaptic dynamics in hippocampal synapses under the conditions of our experiments. Because in-depth analysis of MVR contribution to synaptic dynamics is limited by the lack of a clearly defined relationship between MVR and release probability and because the presence of MVR in our simplified description did not significantly alter model performance, below we used our initial formulation (Eqs. 1,2) to preserve the maximal calculational simplicity of the model.

Postsynaptic mechanisms. Several postsynaptic mechanisms, including receptor saturation and desensitization, have been proposed to play a role in STP in numerous synaptic preparations (Zucker and Regehr, 2002). These postsynaptic mechanisms, however, do not play a significant role in the observed synaptic dynamics in hippocampal synapses under our experimental conditions (Wesseling and Lo, 2002; Klyachko and Stevens, 2006b) and have not been considered in the present study.

Other forms of plasticity. The current model ignores all long-lasting forms of synaptic plasticity, including post-tetanic potentiation, longterm potentiation, and depression, which operate on much longer timescales than were examined in the current study.

\section{Results}

In this study, we considered the interdependencies among STP components and their impact on synaptic release probability within a mechanistically relevant, yet computationally simple model of STP. Our goal was to reproduce the key features of the nonlinear synaptic dynamics during natural stimulus trains, thereby revealing the specific contributions to this dynamics of different STP components. Specifically, the model should be able to reproduce three main characteristics of STP previously observed in excitatory hippocampal synapses (Klyachko and Stevens, 2006a): the high-pass filtering pattern selective for spike discharges associated with place-fields, the nonlinear switch-like pattern in frequency dependence of synaptic response, and the near independence of synaptic response of the discharge temporal characteristics. Our approach to developing such a model thus consisted of three steps: following model formulation, we first "tuned" the model using a series of synaptic responses to constant-frequency trains to determine model parameters. We then used the model to predict synaptic responses to natural spike patterns based solely on the input spike sequence. We determined whether the model can reproduce the nonlinear filtering paradigm observed experimentally in excitatory hippocampal synapses. We then used the model to create experimentally testable predictions, which we verified using recordings in acute hippocampal slices.

\section{Model assumptions and formulations \\ Interdependencies among the STP components and release probability}

The interplay between components of synaptic enhancement, depression, and release probability is determined by considering the synaptic release probability $P_{\text {syn }}$ as a function of vesicle fusion probability $\pi$ and the number of release-competent vesicles $n_{\mathrm{RRP}}$ (Eq. 1). The contribution from facilitation $\Phi$ and augmentation $A$ to release probability is formulated via their contribution to the vesicle fusion probability $\pi$ (Eq. 5). $\Phi$ and $A$ multiply the basal vesicle fusion probability $\lambda$, as follows from the assumption that $\Phi$ and $A$ act independently to lower the energy barrier for vesicle fusion (Eqs. 3-5). The impact of changing release probability on short-term depression is formulated as the reduction in the size of the RRP $n_{\mathrm{RRP}}$ by the amount $P_{\text {syn }}$ (Eq. 14), which represents the average vesicle use per stimulus. Finally, components of enhancement and depression are intrinsically coupled via the contribution of $\Phi$ and $A$ to $P_{\text {syn }}$, which determines the RRP depletion.

\section{Formulations for individual STP components}

Facilitation is formulated similarly to the previous models (Mallart and Martin, 1967; Bertram et al., 1996; Wang, 1999; Dittman et al., 2000; Hempel et al., 2000) assuming that it is associated with some calcium-binding molecule that couples presynaptic residual calcium levels to vesicle fusion, leading to effective facilitation (Eqs. 6-8). Initially, a single component of facilitation was used in the model with the parameters determined from a large set of paired-pulse experiments we previously performed (Klyachko and Stevens, 2006a). Parameters of facilitation $h_{f}$ and $\tau_{f}$ were extracted from the data based on a mono-exponential fit that closely agreed with the data, with a $\tau_{f}$ of $140 \mathrm{~ms}$. We found, however, that the model was unable to reproduce the very rapid increase in synaptic strength at the onset of high-frequency trains or spike bursts during natural stimulation (supplemental Fig. 1, available at www.jneurosci.org as supplemental material), indicating that a second, very rapid component of facilitation must be present to reproduce the rapid response onset. Indeed, two components of facilitation have been observed in many synaptic preparations (Zucker and Regehr, 2002). We therefore performed all analyses with two facilitations present (Eq. 9) (Fig. 1).

Augmentation, the slower component of presynaptic enhancement that is also believed to act by potentiating vesicle fusion (Stevens and Wesseling, 1999), is formulated similarly to facilitation (Eqs. 10-12). The dynamic properties of augmentation have been studied extensively in the NMJ (Magleby and Zengel, 1976; Zengel and Magleby, 1982; Kalkstein and Magleby, 2004), but quantitative data are limited for hippocampal synapses, particularly at near-physiological temperatures. We therefore determined parameters of augmentation evoked by a set of constant-frequency trains at 2-80 Hz, 5-100 stimuli long (Fig. 2). Augmentation parameters were extracted as reported previously (Klyachko and Stevens, 2006b). Note that the time course of augmentation decay is largely independent of the stimulus frequency and the number of stimuli in the train (Fig. $2 B$ ).

The kinetics of recovery from depression in hippocampal synapses at near-physiological temperatures has been measured previously (Klyachko and Stevens, 2006b), giving a time constant of recovery of $\sim 1.2 \mathrm{~s}$ following a 150 -stimuli train at $40 \mathrm{~Hz}$. In agreement with previous studies (Schneggenburger et al., 2002), we found that a single-pool model of recovery from depletion 

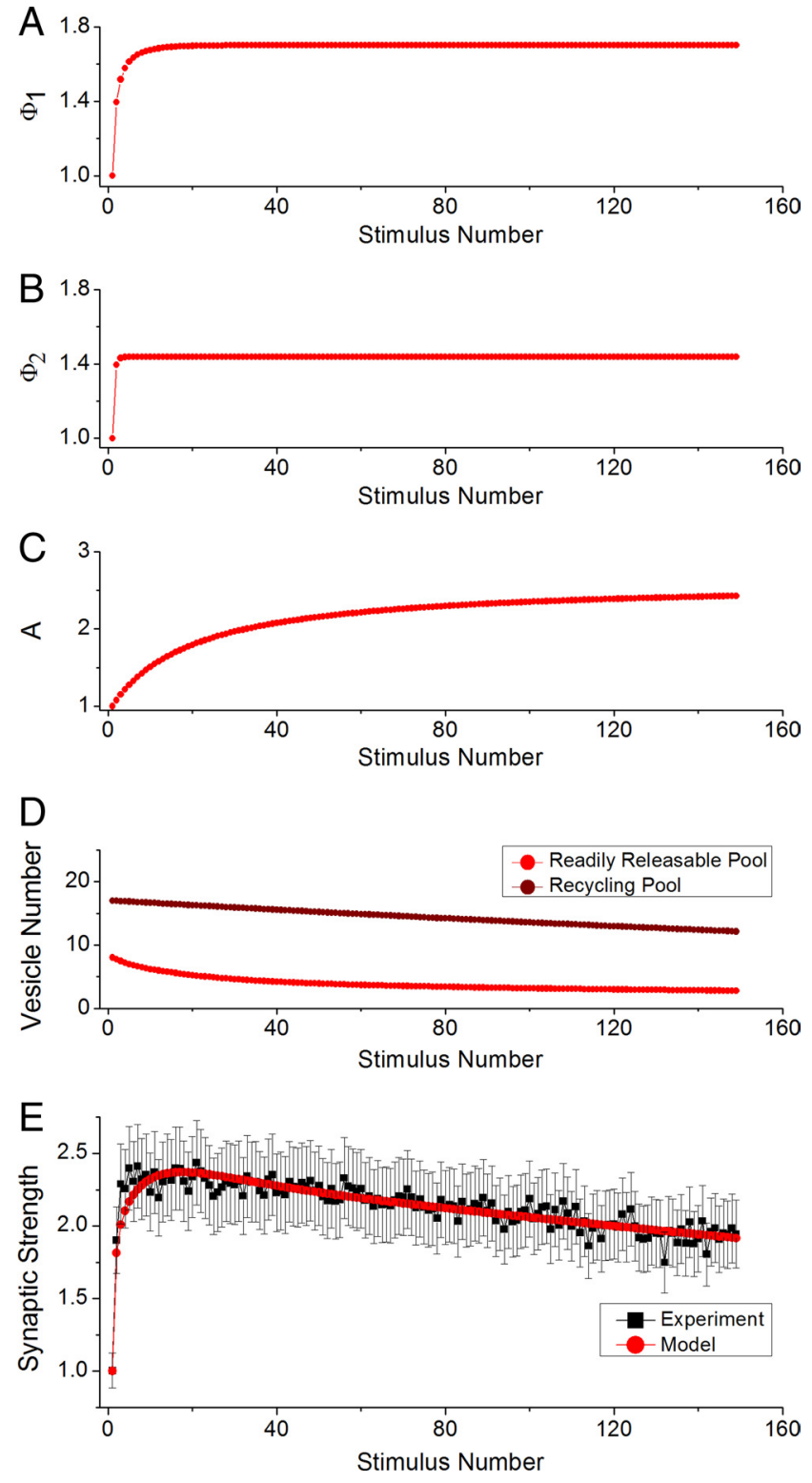

Figure 1. Modeling synaptic dynamics during constant-frequency stimulation. $\boldsymbol{A}-\boldsymbol{D}$, The empirical fit of the model for the experimental data at $40 \mathrm{~Hz}, 150$ stimuli, was calculated by solving the model's nonlinear system of equations using the Levenberg-Marquardt algorithm. $A-C$, The two facilitations, $\Phi_{1}$ with $\tau_{\Phi 1}=140 \mathrm{~ms}$ and $\Phi_{2}$ with $\tau_{\Phi 2}=15 \mathrm{~ms}$, showed rapid onset and saturation $(\boldsymbol{A}, \boldsymbol{B})$, whereas a sustained increase was observed for augmentation $(\boldsymbol{C})$ that had a time constant of $\tau_{\mathrm{A}}=6 \mathrm{~s}$ (Tables 1,2; Fig. 2). The RRP $\left(n_{\text {RRP }}\right)$ and recycling pool $\left(n_{\text {REC }}\right)$ sizes decreased over time from the initial values of 8 and 17 , respectively $(\boldsymbol{D})$. The RRP depleted to a value of $\sim 4$ after 150 stimuli at $40 \mathrm{~Hz}$. $E$, The model fit to the experimental data $(n=12$ cells, from Klyachko and Stevens, 2006a).

does not produce adequate description of the data in that it overestimated vesicle depletion, particularly at higher frequencies of stimulation. We therefore implemented a sequential two-pool model of recovery from depletion (Eqs. 13-15) in which recovery of RRP was aided by replenishment from a second "recycling" pool. We assumed $n_{\mathrm{RRP}}(0)=8$ and $n_{\mathrm{REC}}(0)=17$ vesicles for the initial sizes of the RRP and the recycling pool, respectively, based on published functional and structural measurements in hippocampal synapses (Harris and Sultan, 1995; Murthy et al., 1997; Schikorski and Stevens, 2001). Previous studies suggest that the rate of recovery from depression can be accelerated by elevated calcium levels during synaptic activity (Dittman and Regehr,
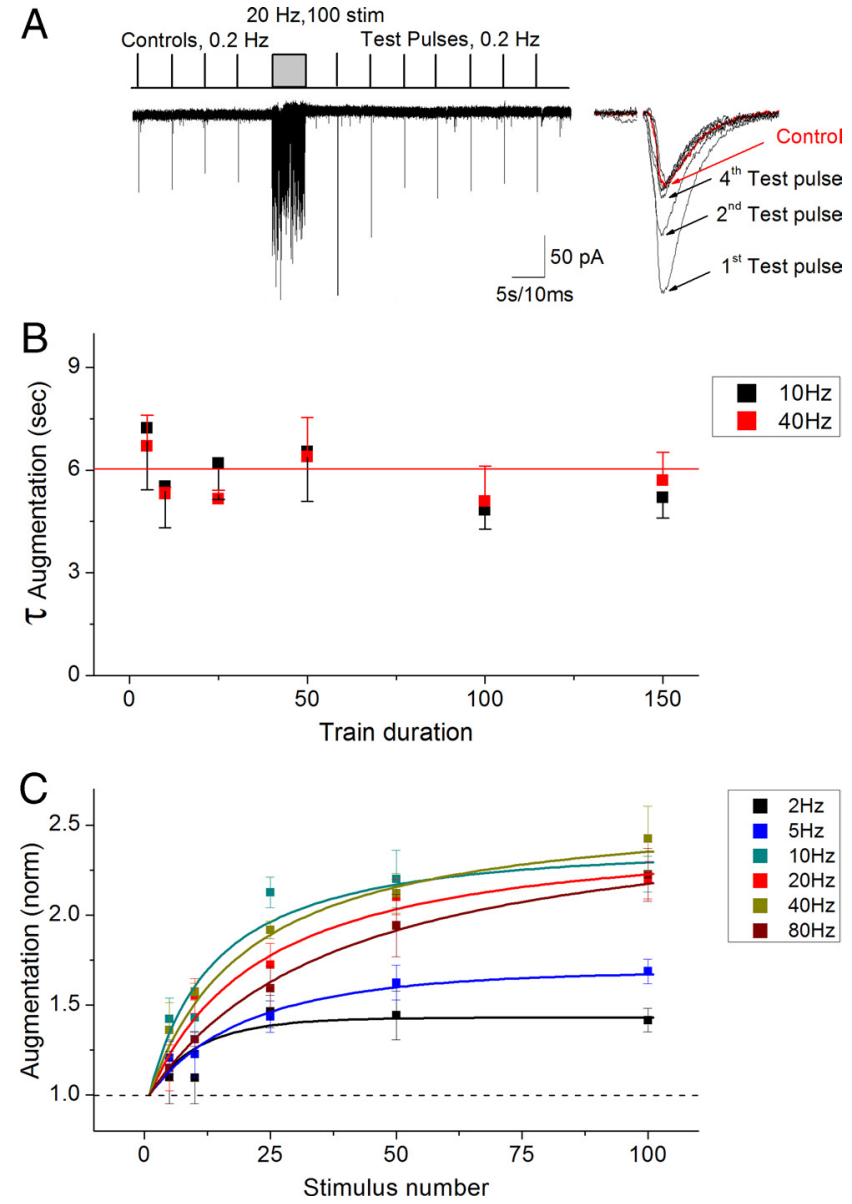

Figure 2. Experimental estimation of augmentation parameters. $\boldsymbol{A}$, An example of an experiment used to determine augmentation parameters. A set of synaptic responses to test pulses at $0.2 \mathrm{~Hz}$ following high-frequency stimulus trains was approximated with a monoexponential fit, and augmentation parameters were extracted as previously described (Klyachko and Stevens, 2006b). The example shown is an average of three trials in the same cell. $\boldsymbol{B}$, The decay time constant of augmentation was measured for a set of stimulus trains of different durations (5-150 stimuli) at 10 and $40 \mathrm{~Hz}(n=6-12$ cells). A linear fit showed that augmentation time constant $\tau_{\alpha}=6 \mathrm{~s}$ is largely independent of the train duration and frequency. C, The $h_{\alpha}$ parameter of augmentation was determined by solving the nonlinear equations (Eqs. 10, 12) using the experimentally determined amplitude of augmentation measured for a set of $5-100$ stimuli trains at $2-80 \mathrm{~Hz}$ as described above ( $n=6-9$ cells). For the $\mathrm{ISIs}<25 \mathrm{~ms}$ and $>500 \mathrm{~ms}$, the $h_{\alpha}$ parameter of augmentation was determined by linear extrapolation because of the large variability in experimental values at these ISIs. Other parameters of augmentation were set as described in Tables 1 and 2.

1998; Sakaba and Neher, 2001). Because the extent of calciumdependent recovery from depletion varies greatly among different synapses (Neher and Sakaba, 2008) and because it is not clear which of the refilling rates in our model might be accelerated by calcium, we did not explicitly account for the calcium-dependent recovery but kept the refilling time constants as free parameters. Indeed, our analysis showed an acceleration of the RRP refilling rate with increasing stimulus frequency (Tables 1,2), which presumably correlates with the increase in intraterminal calcium concentration.

\section{Model tuning by fitting synaptic responses to constant-frequency trains}

To be able to predict synaptic behavior during natural patterns of stimulation, we first determined the model parameters and constants not available from the literature by fitting the model to a set of constant-frequency train responses recorded from CA1 pyra- 
Table 1. Constants

\begin{tabular}{lcll}
\hline Parameter & Values & Description & Reference \\
\hline$\pi$ & 0.035 & Basal vesicle fusion probability & (Murthy et al., 1997) \\
$n_{R R P} \mid t=0$ & 8 & Readily releasable pool size & (Schikorski and Stevens, 2001) \\
$n_{R E C} \mid t=0$ & 17 & Recycling pool size & (Schikorski and Stevens, 2001) \\
$\tau_{f 1}$ & $140 \mathrm{~ms}$ & Slower facilitation time constant & $\begin{array}{l}\text { (Klyachko and Stevens, } \\
\text { 2006a,b) }\end{array}$ \\
$\tau_{f 2}$ & $15 \mathrm{~ms}$ & Faster facilitation time constant & (Zucker and Regehr, 2002) \\
$\tau_{\alpha}$ & $6000 \mathrm{~ms}$ & Augmentation time constant & (Klyachko and Stevens, \\
& & & 2006a,b) \\
$\tau_{D 1}$ & $1200 \mathrm{~ms}$ & RRP recovery time constant & (Klyachko and Stevens, \\
& & & 2006a,b) \\
$\eta_{1}$ & 1.21 & Constant determining max. $\Phi_{1}$ & \\
$\eta_{2}$ & 1.21 & Constant determining max. $\Phi_{2}$ & \\
$\mu$ & 0.59 & Constant determining max. A & \\
$\theta$ & 1 & Cooperativity factor for $\Phi_{1} / \Phi_{2} / \mathrm{A}$ & \\
& &
\end{tabular}

Table 2. Variable ranges from experimental data

\begin{tabular}{lccccl}
\hline & \multicolumn{3}{l}{ Frequency in $\mathrm{Hz}$} & \\
\cline { 2 - 5 } Parameter & 2 & 10 & 20 & 40 & Source \\
\hline$h_{\alpha}$ & 0.0462 & 0.1113 & 0.0653 & 0.0818 & Computed from augmentation data \\
$h_{\mathrm{f} 1}, h_{\mathrm{f} 2}$ & 0.1032 & 0.4332 & 0.5609 & 0.7560 & Computed from paired-pulse data \\
$\tau_{D 2}(\mathrm{~ms})$ & 258.68 & 52.91 & 17.94 & 8.85 & Computed from const. frequency fits \\
$\tau_{D 3}(\mathrm{~s})$ & 195.05 & 9.65 & 19.06 & 10.96 & Computed from const. frequency fits \\
\hline
\end{tabular}

midal cells (data from Klyachko and Stevens, 2006a) in whole-cell configuration in response to stimulation of Schaffer Collaterals (Figs. 1, 3). Note that both components of facilitation saturated rapidly and contributed little to synaptic dynamics after $\sim 5$ consecutive stimuli, whereas augmentation continued to increase throughout the stimulus train, although at a reducing rate. This analysis produced the remaining parameters and constants of the model that were not available from the literature and are summarized in Tables 1 and 2. Overall, the model effectively captured all of the main features of synaptic responses to constant-frequency stimulation, mainly a rapid increase in synaptic strength at the onset of the stimulus train, saturation of synaptic enhancement with frequency above $\sim 10 \mathrm{~Hz}$, and a gradual increase in apparent depression with frequency (Fig. 3).

\section{Prediction of synaptic responses to natural stimulus patterns}

If the model correctly captures the basic principles of synaptic dynamics, we expect that, once tuned on constant-frequency data, the model should be able to predict synaptic responses to natural patterns of stimulation. The model prediction for synaptic response to a natural spike train was calculated solely based on the timing of the input stimuli and had no free parameters (Fig. $4 A, B)$. We found that the model accurately reproduced the key characteristics of synaptic dynamics during natural spike trains, such as selective high-pass filtering of spike discharges associated with place-fields (Klyachko and Stevens, 2006a) (Fig. 4A,B). The model further correctly captured the rapid onset of synaptic response changes at the beginning of the spike bursts and rapid saturation of synaptic strength during the bursts. Note, however, that the model slightly but consistently overpredicted the synaptic strength during natural stimulus trains, particularly following the end of high-frequency bursts (Fig. 4A, B; supplemental Fig. 3, available at www.jneurosci.org as supplemental material). This difference may arise from the model's underestimation of shortterm depression, because we did not take into account additional depletion-independent processes since their contributions in hippocampal synapses are not well established. To quantitatively
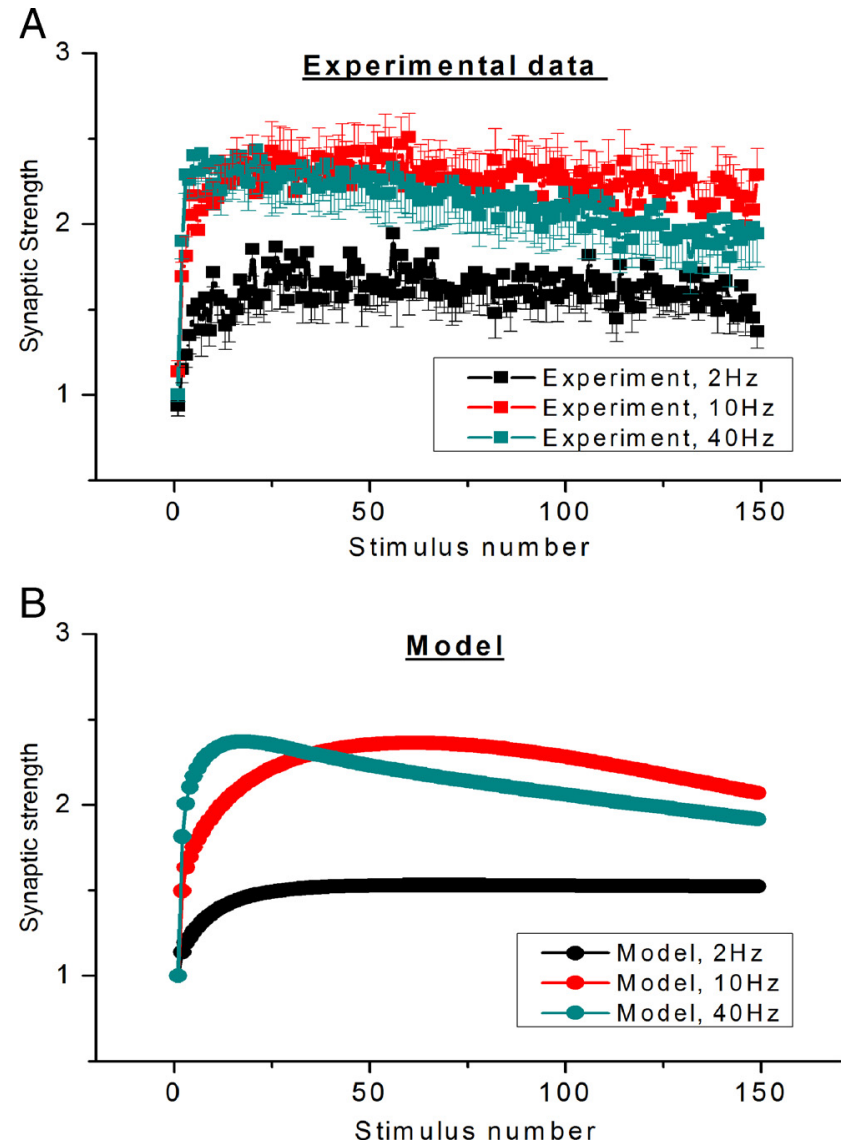

Figure 3. The model tuning using constant-frequency data. $A, B$, Comparison between the experimental data $(\boldsymbol{A})(n=9-12$ cells) and the corresponding model fits $(\boldsymbol{B})$ at 2,10 , and $40 \mathrm{~Hz}$, 150 stimuli. The model successfully captured different characteristics of synaptic responses to constant-frequency stimulation. These fits were used to determine parameters of the model not available from the previous experimental work (Tables 1, 2).

compare the model prediction and the experimental results, we used correlation analysis in which changes in synaptic strength in the model and experimental data were plotted point by point versus each other for each stimulus in the train (Fig. 4C). Linear regression gives an average value of $R=0.88$, suggesting a strong similarity between the model prediction and experiment. This high degree of similarity between computed and experimentally observed synaptic dynamics was reproducible across different natural spike patterns (Fig. 4C).

The model also qualitatively reproduced the switch-like frequency dependence of synaptic response during natural stimulus trains (Fig. 5A; supplemental Fig. 3, available at www.jneurosci. org as supplemental material). Similarly to experimental results, the model showed a rapid transition between the basal (at low frequency) and elevated (at high frequency) synaptic strength levels as well as rapid saturation of the response with input frequency. The elevated strength level exhibited by the model was similar to that observed in the experiment, as determined by a Boltzmann function fit (2.37 and 2.26, respectively). An interesting feature of this filtering pattern that we previously observed is that synaptic response during natural stimulus trains was largely independent of the discharge frequency and duration (Klyachko and Stevens, 2006a), suggesting that nearly all place-field discharges are processed independently of the temporal pattern within the discharges. This feature was closely reproduced by the model for both discharge frequency and duration (Fig. $5 B, C$ ). 

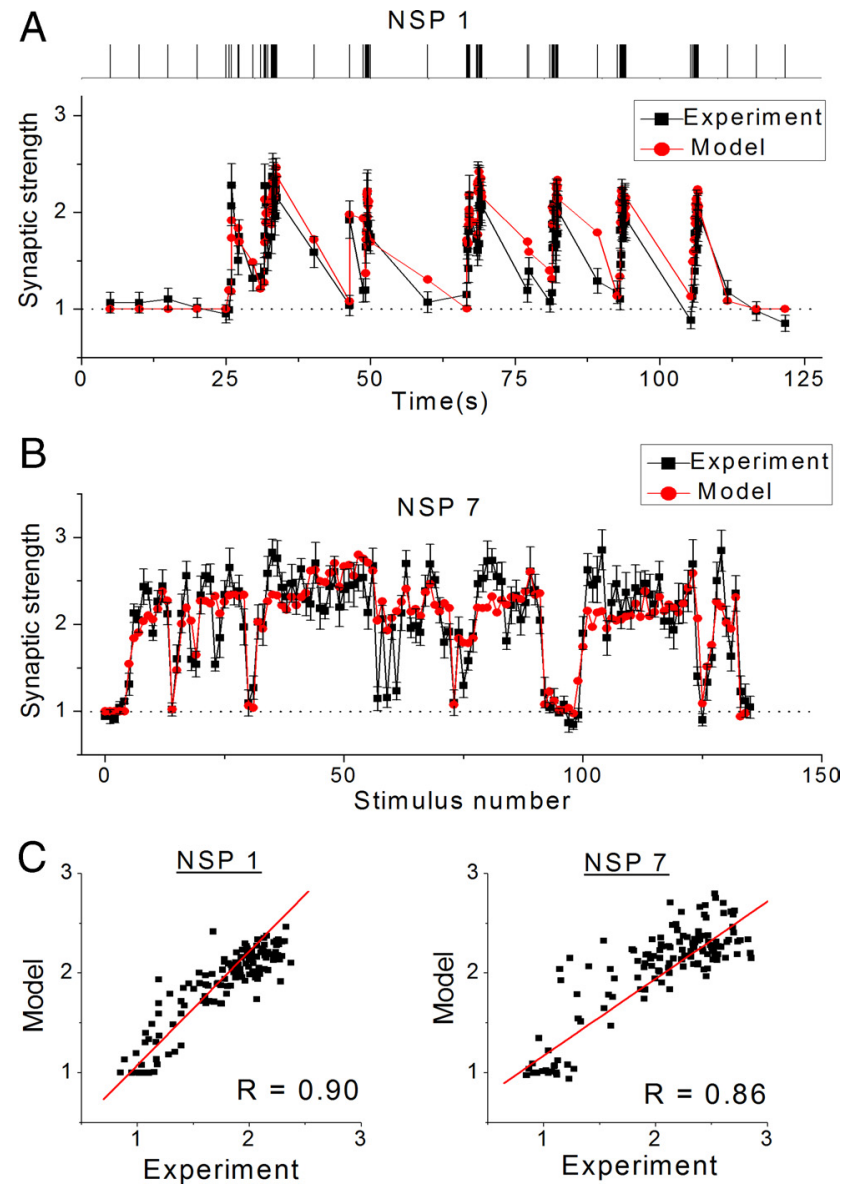

Figure 4. Prediction of synaptic dynamics during natural stimulus trains. $A$, Relative changes in synaptic strength (black) (experimental data from Klyachko and Stevens, 2006a) during the natural spike train (NSP1) shown above and the model prediction exclusively based on interspike intervals (red). No free parameters or fitting algorithms were used to calculate model prediction of the synaptic responses during natural stimulation. $\boldsymbol{B}$, Same as $\boldsymbol{A}$ but plotted against stimulus number and for a different natural spike train (NSP7). Note that the model slightly but consistently overpredicts the synaptic strength during, and particularly after, highfrequency bursts. $\boldsymbol{C}$, The Pearson's correlation coefficient was measured to estimate correlation between the model prediction and the experimental data. Two examples of this correlation analysis for two different natural stimulus trains are shown.

Together these results demonstrate that this simple model correctly captures the main aspects of synaptic dynamics during natural stimulus patterns in hippocampal synapses.

\section{Specific roles of different forms of STP in synaptic dynamics}

One of the main aims of developing a simple mechanistic model of STP is to reveal the contributions of individual STP components to overall complex synaptic dynamics. This problem has been difficult to address so far because of the lack of experimental approaches to selectively eliminate one of the components of STP without affecting the others. For example, application of $\mathrm{Sr}^{2+}$ or $\mathrm{Ba}^{2+}$ ions has been shown to selectively suppress facilitation or augmentation in the NMJ (Zengel and Magleby, 1977). This treatment, however, is not applicable to the hippocampal synapses where $\mathrm{Ba}^{2+}$ application leads to strong depression (Klyachko and Stevens, 2006b) and $\mathrm{Sr}^{2+}$ causes a shift from synchronous to asynchronous release (Goda and Stevens, 1994). To address this question computationally, we removed contributions of individual components from the model one by one and studied the resulting changes in synaptic strength.

Facilitation $\Phi$ or augmentation $A$ were silenced by making $\mathrm{h}_{\mathrm{f} 1}, \mathrm{~h}_{\mathrm{f} 2}=0$ or $\mathrm{h}_{\alpha}=0$, respectively, while synaptic depression was
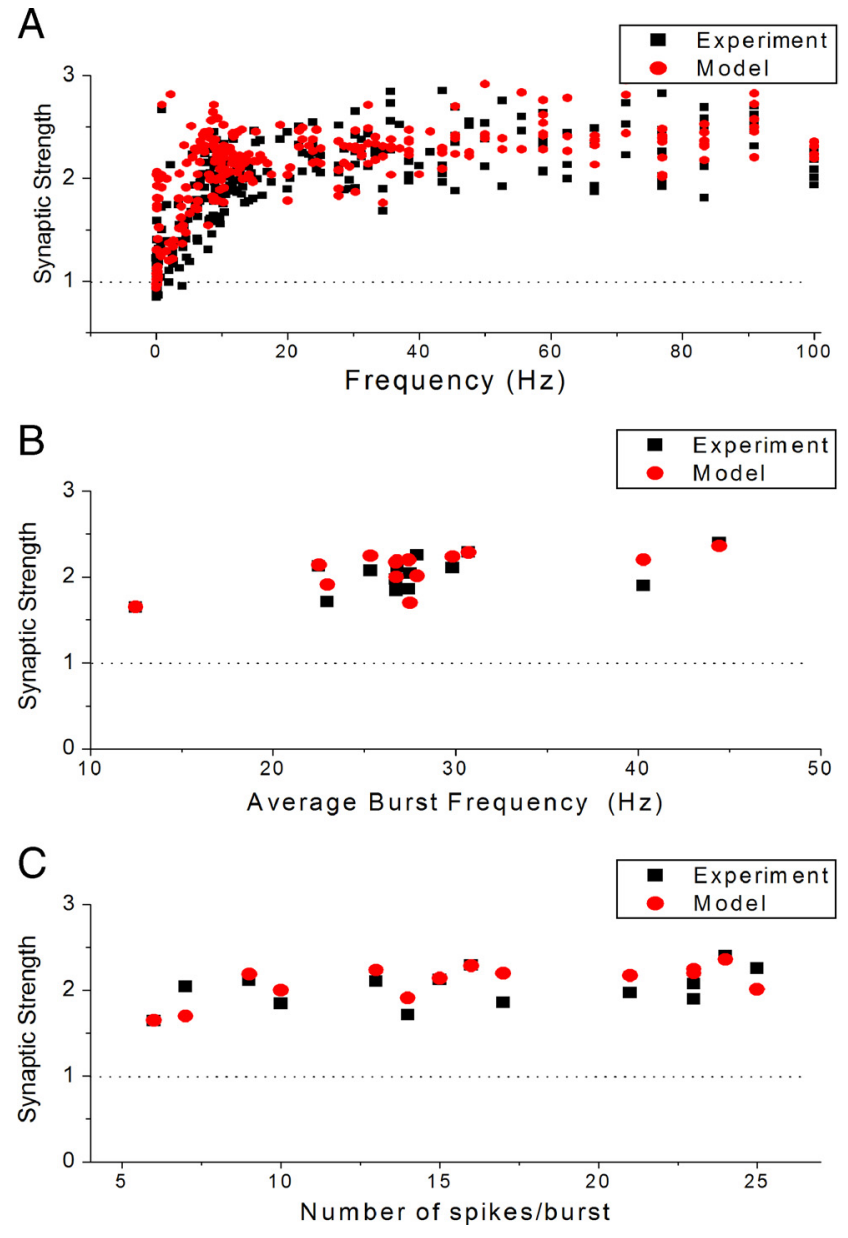

Figure 5. Model prediction of synaptic filtering paradigm during natural stimulus trains. A, A plot of synaptic strength versus instantaneous input frequency for the experimental data (black) (Klyachko and Stevens, 2006a) and model (red). The model correctly captured the highpass filtering nature of STP in hippocampal synapses. $\boldsymbol{B}, \boldsymbol{C}$, Model correctly reproduced the experimental observation (Klyachko and Stevens, 2006a) that synaptic strength during placefield discharges is largely independent of discharge average frequency $(\boldsymbol{B})$ and the number of spikes in the discharge (C).

removed by making $n_{\mathrm{RRP}}=n_{\mathrm{RRP}}(0)=$ constant. We found that removal of facilitation led to a much slower and more gradual initial increase in synaptic strength, and this effect became more apparent as frequency increased (Fig. 6). In contrast, in the absence of augmentation, synaptic strength increased rapidly but could not be sustained at the experimentally observed elevated levels in the face of increasing depression. Finally, in the absence of depression, synaptic strength increased rapidly at the onset of stimulation, but, unlike experimental data, continued to increase at a slower rate throughout the stimulus train. This analysis showed that facilitation is required for the rapid increase in synaptic strength at the onset of high-frequency input, whereas augmentation is essential to sustain the experimentally observed levels of release during high-frequency activity. Depression, on the other hand, controls the dynamic range of this synaptic filter and provides rapid response saturation, thus preventing runaway synaptic enhancement. The latter model prediction regarding the role of depression provides an opportunity to experimentally verify the model performance, because, unlike facilitation and augmentation, levels of synaptic depression are amenable to experimental manipulation. 

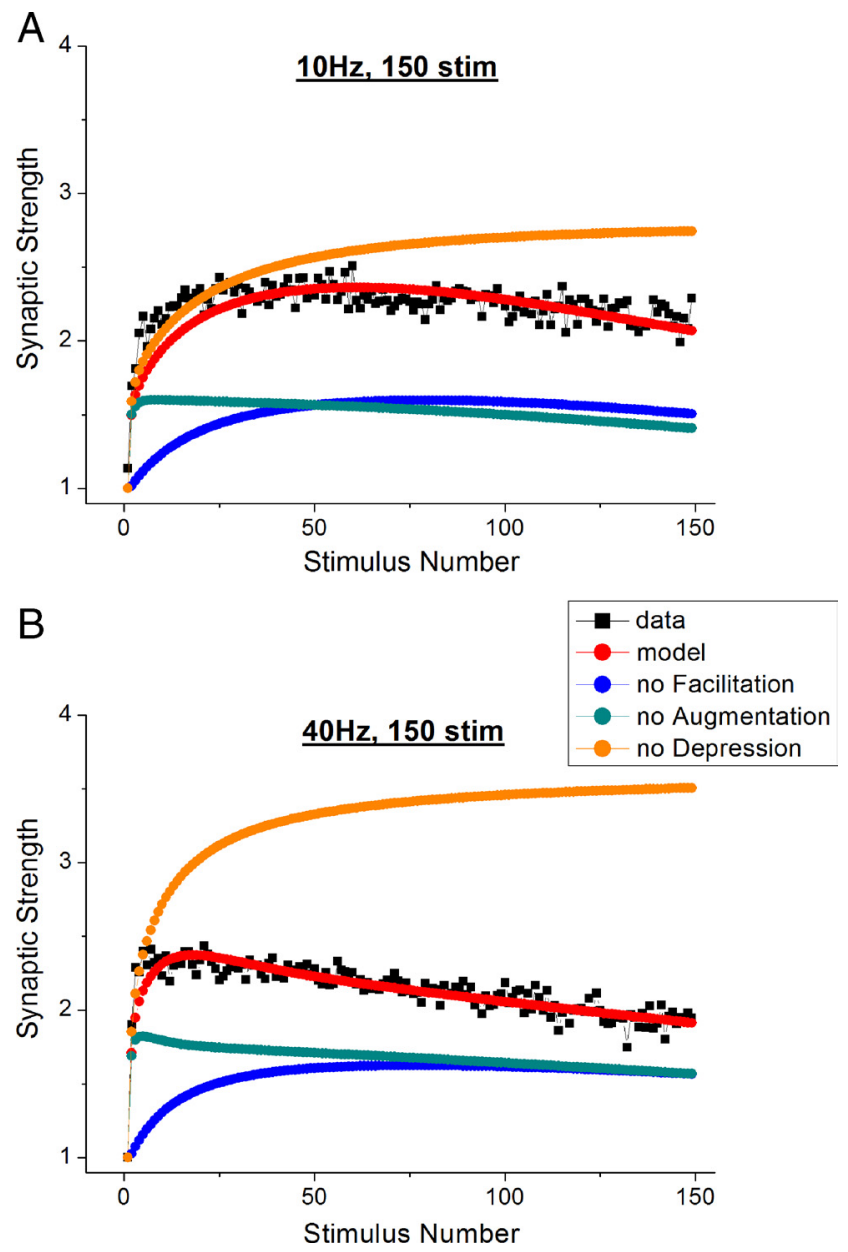

Figure 6. The roles of individual STP components in synaptic dynamics. $A, B$, The contributions of different STP components were evaluated by eliminating individual components from the model. Two examples of this analysis for constant-frequency trains at 10 and $40 \mathrm{~Hz}$ are shown in $\boldsymbol{A}$ and $\boldsymbol{B}$, respectively. The absence of facilitation resulted in a much slower rise in synaptic strength at the onset of stimulation than that observed in the experiment. Removal of augmentation prevented the model from reaching the level of increase in synaptic strength observed experimentally. Removal of depression prevented rapid saturation of synaptic strength changes observed in the experiment.

Prediction of calcium-dependent changes in synaptic filtering paradigm and its test in hippocampal slices

To validate the model's prediction, we took advantage of the fact that lowering the extracellular calcium concentration reduces the basal vesicle fusion probability $\lambda$, thus reducing vesicle use and depletion (Zucker and Regehr, 2002). The model predicts that under these conditions of reduced depression, synaptic response continuously increases during high-frequency stimulation (Fig. 6). The model also predicts large calcium-dependent changes in the synaptic filtering pattern during natural stimulus trains; synaptic strength during discharges at reduced extracellular calcium is expected to not rapidly saturate like that observed at standard calcium concentration but rather to continue to increase, reaching much more elevated strength levels.

To computationally reproduce the decrease in vesicle depletion at reduced extracellular calcium, we modified a single model parameter, the basal vesicle fusion probability $\lambda$, which was reduced to 0.0002 (Fig. 7A). The synaptic dynamics during stimulus trains was then determined without free parameters, solely based on the timing of stimuli during the train. As predicted, the reduced $\lambda$ led to a much larger continuous increase in synaptic strength during constant-frequency trains (Fig. $7 B$ ), similar to the situation in which depression was removed in our deletion analysis (Fig. 6). The model produced a similar effect when natural stimulus patterns were used as the model input (Fig. 7C), leading to large changes in the synaptic filtering pattern (Fig. $7 D$ ). We verified these predictions using whole-cell recordings in CA1 pyramidal cells in hippocampal slices at $0.7 \mathrm{~mm}$ extracellular calcium. Synaptic strength during constant frequency (Fig. $7 F$ ) or natural spike trains (Fig. $7 G$ ) qualitatively showed the same behavior as predicted by the model. Note that under these conditions, changes in synaptic dynamics were attributed mostly to reduced vesicle depletion, because facilitation and augmentation changed relatively little (Fig. $7 E$ ). These results indicate that based on changes in a single nonvariable parameter $\lambda$, the model correctly predicted the calcium dependence of the synaptic filtering paradigm in hippocampal synapses (Fig. $7 D, H$ ). These results further suggest that short-term depression in part controls the dynamic range of the synaptic filter, thereby allowing highfrequency discharges to be recognized with high fidelity (Fig. 4) yet causing rapid saturation of synaptic strength during bursts to prevent runaway enhancement (Figs. 6, 7).

\section{Discussion}

In this study, we explored a mechanistically relevant model of STP to better understand the underlying mechanisms of highly nonlinear synaptic dynamics observed in excitatory hippocampal synapses during natural stimulus patterns (Klyachko and Stevens, 2006a). The model considers interrelationships among four major forms of STP in hippocampal synapses, two components of facilitation, augmentation, and depression, and describes their impact on synaptic release probability. The interactions among calcium-dependent forms of presynaptic enhancement are formulated assuming that they act independently to lower the energy barrier for vesicle fusion, leading to increased probability of release. The impact of short-term enhancement on synaptic depression is formulated in terms of vesicle depletion by taking into account that elevated release probability leads to increased vesicle use. Finally, the basic probability theory is used to define the impact of these STP components on synaptic release probability. Once tuned, the model captures all key features of synaptic dynamics during natural spike trains without free parameters. The model further explains the specific roles of individual STP components in shaping synaptic dynamics. Finally, the model predicts strong calcium dependence of synaptic filtering behavior, a prediction we experimentally verified in hippocampal slices. Our approach thus offers a useful framework to further investigate the role of STP in synaptic computations.

\section{Relationship to other STP models}

Our description of individual STP components is based in part on previous formulations but departs from them in several ways. Facilitation is treated similarly to that described by (Mallart and Martin, 1967; Bertram et al., 1996; Dittman et al., 2000; Hempel et al., 2000). This treatment emphasizes rapid saturation of facilitation as experimentally observed in hippocampal synapses (Dobrunz and Stevens, 1997). This is in contrast to quantitative description of facilitation in the NMJ, in which saturation of facilitation was not necessary to account for experimental data (Magleby and Zengel, 1982; Zengel and Magleby, 1982), possibly reflecting differences in the initial release probability in the two experimental systems.

Augmentation is treated similarly to facilitation, based on the similarity of these STP components in hippocampal synapses 
(Stevens and Wesseling, 1999). Our description, however, differs from previous models developed for the NMJ because of the apparent quantitative differences in the dynamic properties of augmentation between the two types of synapses (Magleby and Zengel, 1982; Zengel and Magleby, 1982). In the NMJ, augmentation was found to undergo an accelerating increase during repetitive stimulation under conditions of low release probability. In hippocampal synapses, augmentation exhibits saturating behavior with increasing train frequency and duration (Fig. 2). These differences could arise from changes in dynamic properties of augmentation at higher release probabilities in hippocampal synapses or perhaps from the intrinsic differences in augmentation between the NMJ and hippocampal synapses.

Our treatment of interactions among components of enhancement assumes that they act independently to lower the energy barrier for vesicle fusion. We further assumed that these components are mediated by different calcium-binding molecules, which translate elevation in residual calcium levels into effective facilitation/augmentation. Although the identity of such calcium-binding molecules has been debated, several studies have suggested that a priming factor Munc-13-2 regulated by calmodulin may serve as a molecular determinant of augmentation (Rosenmund et al., 2002; Junge et al., 2004), and the Neuronal Calcium Sensor-1 has been proposed as a candidate for facilitation (Sippy et al., 2003). Alternative models considering calcium buffer saturation as the underlying mechanism of facilitation have been proposed and successfully verified in several experimental systems (Klingauf and Neher, 1997; Blatow et al., 2003; Matveev et al., 2004) but do not seem to fully account for facilitation in other synapses (Pan and Zucker, 2009), including the excitatory hippocampal ones (Sippy et al., 2003). The identity of the molecular determinants of facilitation and augmentation thus remains unclear, and our model assumption of independent action by facilitation and augmentation will require future verification.

Short-term depression is modeled as vesicle depletion, similarly to the approaches previously proposed (Kusano and Landau, 1975; Heinemann et al., 1993; Weis et al., 1999). We chose to use a sequential rather that the parallel model of vesicle pool refilling, because it better matches the experimentally observed vesicle release dynamics in hippocampal synapses (Dobrunz and Stevens, 1997; Stevens and Wesseling, 1998; Sara et al., 2002) and because
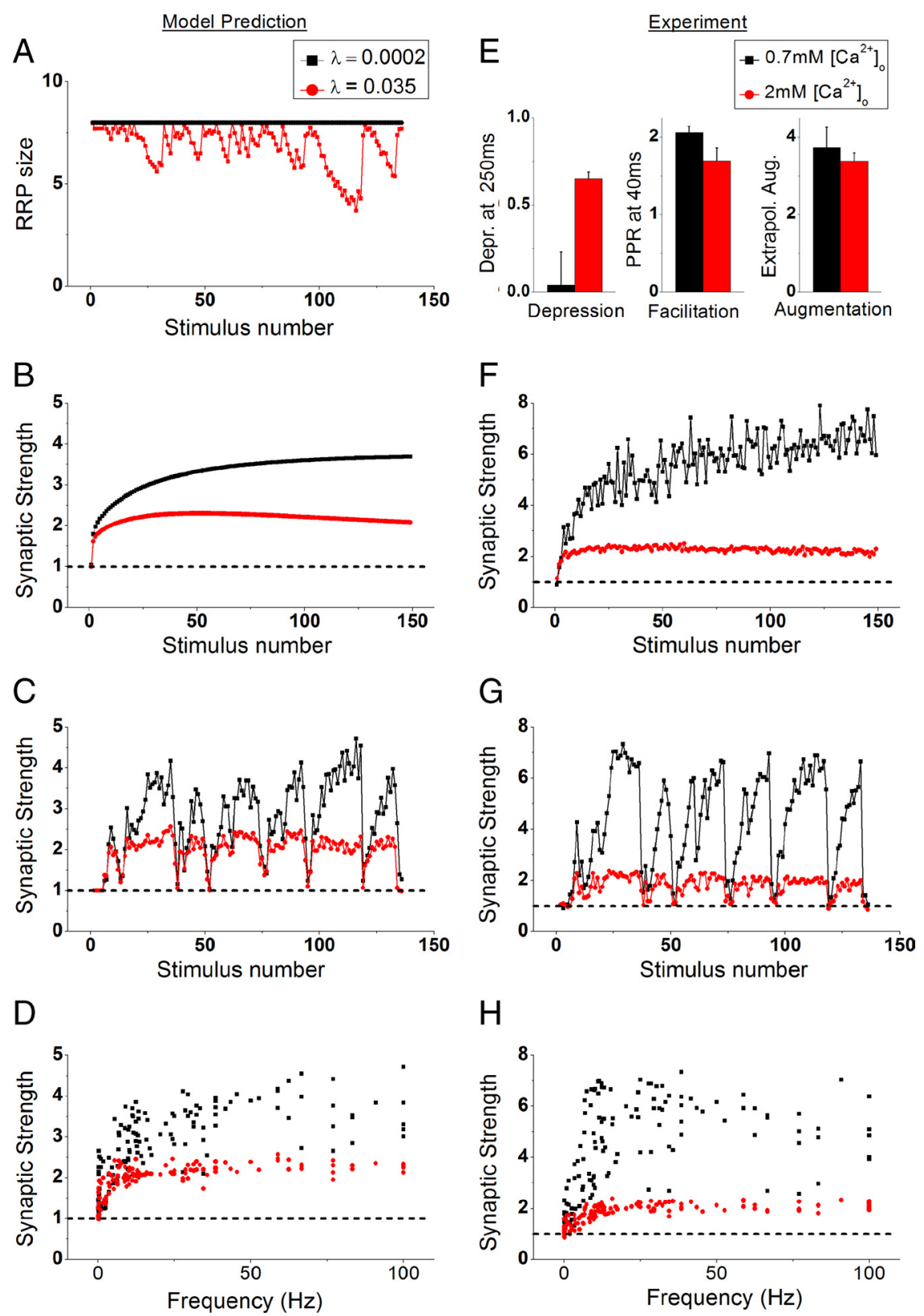

Figure 7. Experimental verification of the model prediction. $\boldsymbol{A}-\boldsymbol{D}$, The model predicts that under the conditions of reduced vesicle use, when depletion is nearly absent $(\boldsymbol{A})$, synaptic strength does not saturate rapidly but rather continues to increase with stimulation, similarly to that in the absence of depression seen in Figure 6 . The model prediction for the changes in synaptic dynamics under conditions of reduced depletion (black) versus normal conditions (red) is shown for a constant-frequency ( $40 \mathrm{~Hz}$, 150 -stimuli) train ( $\boldsymbol{B}$ ) and a natural stimulus train (C). The resulting changes in the synaptic filtering paradigm are shown in $\boldsymbol{D}$. The reduction in vesicle depletion was implemented by changing a single model parameter, the basal vesicular release probability $\lambda$. No fitting or free parameters were used in calculating the model prediction. $\boldsymbol{E}-\boldsymbol{H}$, The conditions of reduced depletion were experimentally reproduced by lowering the extracellular calcium concentration from 2 to $0.7 \mathrm{~mm}$. $\boldsymbol{E}$, This treatment resulted in greatly reduced depression, while facilitation and augmentation were affected relatively little $(n=8)$. Parameters of STP components were determined from whole-cell recordings in acute hippocampal slices as previously described (Klyachko and Stevens, 2006b). F, G, The experimentally measured changes in synaptic strength at normal ( $n=12$, red) and reduced ( $n=8$, black) calcium concentrations are shown for the same constant-frequency stimulus train $(\boldsymbol{F})$ and a natural stimulus train $(\boldsymbol{G})$ as that used in $\boldsymbol{B}, \boldsymbol{C}$. The resulting changes in synaptic filtering paradigm are shown in $(\boldsymbol{H})$.

structural data suggest the presence of a single active zone in the majority of these synapses (Schikorski and Stevens, 1997). While several lines of evidence indicate that vesicle depletion is a dominant contributor to rapid depression in hippocampal synapses (Dobrunz and Stevens, 1997; Sara et al., 2002; Fernández-Alfonso and Ryan, 2004), other depletion-independent mechanisms may 
contribute to reduced synaptic strength during high-frequency activity. Indeed, refractiveness of release cites, calcium channel inactivation, and calcium-induced inhibition of calcium channels have been proposed to drive synaptic depression in various types of synapses (Schneggenburger et al., 2002; Zucker and Regehr, 2002). The contributions of these processes to the hippocampal short-term depression, however, remain unclear. The refractiveness of release sites, for example, closely describes short-term depression in cerebellar synapses (Dittman et al., 2000) but appears to strongly overestimate the extent of depression in hippocampal synapses by predicting nearly maximal depression after $\sim 3$ stimuli at $50 \mathrm{~Hz}$, whereas we did not detect significant depression after the same stimulus under our experimental conditions (data not shown). Further studies are needed to establish contributions of depletion-independent mechanisms to hippocampal short-term depression to be able to incorporate them into more detailed models of STP.

\section{The roles of STP components in synaptic dynamics}

One of the fundamental questions in understanding synaptic computations is the specific roles that individual forms of STP play in synaptic dynamics. Several different computations have been attributed to synaptic depression, particularly in synapses where depression represents a single dominant form of STP (Abbott et al., 1997; Chance et al., 1998; Chung et al., 2002; Cook et al., 2003). The contributions of various STP components to synaptic computations have been more difficult to determine in synapses expressing multiple overlapping and interacting forms of STP (MacLeod et al., 2007). We used our modeling approach to examine the roles of STP components in synaptic dynamics based on elimination analysis (Fig. 6). Our results indicate that facilitation is required for the rapid increase in synaptic strength at the onset of high-frequency input. Facilitation thus controls rapid switching of synaptic strength from the basal level during low activity to elevated levels during high-frequency spike bursts. Removal of augmentation suggests that it is a major component of STP that counterbalances synaptic depression to sustain neurotransmitter release during long-lasting spike discharges. Indeed, earlier models of presynaptic dynamics that did not include augmentation (Dobrunz and Stevens, 1997; Dittman et al., 2000) exhibited only transient enhancement (for 2-5 stimuli) rapidly followed by dominating depression. Augmentation is thus essential to account for the sustained synaptic enhancement observed in hippocampal synapses for tens of stimuli at high input frequencies under near-physiological conditions (Fig. 3) (Klyachko and Stevens, 2006b). A similar function for augmentation was also previously proposed for the NMJ (Kalkstein and Magleby, 2004) and hippocampal synapses (Garcia-Perez and Wesseling, 2008). Removal of depression suggests its role in synaptic computations as a mechanism for a rapid synaptic strength control. Unlike the dynamic gain-control mechanism proposed for cortical synapses (Abbott et al., 1997), where dominant depression scales synaptic inputs to allow equal percentage rate changes for afferents firing at different frequencies, masked depression in excitatory hippocampal synapses controls the dynamic range of the synaptic filter, working to rapidly saturate changes in synaptic strength.

\section{Nonlinear synaptic dynamics during natural stimulus trains}

A key feature of hippocampal STP is the strong nonlinearity of the synaptic filtering paradigm during physiologically relevant stimulus patterns (Klyachko and Stevens, 2006a). This filtering paradigm is characterized by a rapid transition between basal and elevated synaptic gain levels and the near frequency independence of synaptic gain above the transition frequency. By accounting for the interdependencies among STP components and release probability, our model captures these key features of the synaptic filtering pattern without free parameters (Figs. 4, 5). Our analysis suggests that while facilitation provides a rapid onset of synaptic strength changes and then rapidly saturates (Fig. 1), the balance between augmentation and depression driven by their interdependency with the release probability provides a rapid leveling of synaptic gain changes. This leads to the nearfrequency independence of synaptic strength at all frequencies relevant to place-field discharges. Indeed, the model prediction confirmed by our experimental recordings (Fig. 7) shows that disrupting the balance among the components by, for example, reducing the initial vesicle fusion probability markedly slows response saturation during high-frequency discharges, thereby causing an excessive increase in synaptic strength. It is tempting to speculate that a relatively modest increase in synaptic strength during spike discharges under normal conditions is tuned to provide a balance between a sufficient dynamic range of the STP filter and ability to rapidly restore the basal state with minimal resource consumption. Future studies will be needed to determine the significance of this tuning and uncover other regulatory factors that may control the dynamic range and tuning of the STP filter during computations.

\section{References}

Abbott LF, Varela JA, Sen K, Nelson SB (1997) Synaptic depression and cortical gain control. Science 275:220-224.

Auger C, Kondo S, Marty A (1998) Multivesicular release at single functional synaptic sites in cerebellar stellate and basket cells. J Neurosci 18:4532-4547.

Bertram R, Sherman A, Stanley EF (1996) Single-domain/bound calcium hypothesis of transmitter release and facilitation. J Neurophysiol 75:1919-1931.

Blatow M, Caputi A, Burnashev N, Monyer H, Rozov A (2003) Ca2 + buffer saturation underlies paired pulse facilitation in calbindin-D28kcontaining terminals. Neuron 38:79-88.

Chance FS, Nelson SB, Abbott LF (1998) Synaptic depression and the temporal response characteristics of V1 cells. J Neurosci 18:4785-4799.

Christie JM, Jahr CE (2006) Multivesicular release at Schaffer collateralCA1 hippocampal synapses. J Neurosci 26:210-216.

Chung S, Li X, Nelson SB (2002) Short-term depression at thalamocortical synapses contributes to rapid adaptation of cortical sensory responses in vivo. Neuron 34:437-446.

Cook DL, Schwindt PC, Grande LA, Spain WJ (2003) Synaptic depression in the localization of sound. Nature 421:66-70.

Dittman JS, Regehr WG (1998) Calcium dependence and recovery kinetics of presynaptic depression at the climbing fiber to Purkinje cell synapse. J Neurosci 18:6147-6162.

Dittman JS, Kreitzer AC, Regehr WG (2000) Interplay between facilitation, depression, and residual calcium at three presynaptic terminals. J Neurosci 20:1374-1385.

Dobrunz LE, Stevens CF (1997) Heterogeneity of release probability, facilitation, and depletion at central synapses. Neuron 18:995-1008.

Fenton AA, Muller RU (1998) Place cell discharge is extremely variable during individual passes of the rat through the firing field. Proc Natl Acad Sci U S A 95:3182-3187.

Fernández-Alfonso T, Ryan TA (2004) The kinetics of synaptic vesicle pool depletion at CNS synaptic terminals. Neuron 41:943-953.

Foster KA, Crowley JJ, Regehr WG (2005) The influence of multivesicular release and postsynaptic receptor saturation on transmission at granule cell to Purkinje cell synapses. J Neurosci 25:11655-11665.

Garcia-Perez E, Wesseling JF (2008) Augmentation controls the fast rebound from depression at excitatory hippocampal synapses. J Neurophysiol 99:1770-1786.

Geppert M, Goda Y, Hammer RE, Li C, Rosahl TW, Stevens CF, Südhof TC (1994) Synaptotagmin I: a major Ca2 + sensor for transmitter release at a central synapse. Cell 79:717-727. 
Goda Y, Stevens CF (1994) Two components of transmitter release at a central synapses. Proc Natl Acad Sci U S A 91:12942-12946.

Guzowski JF, Knierim JJ, Moser EI (2004) Ensemble dynamics of hippocampal regions CA3 and CA1. Neuron 44:581-584.

Harris KM, Sultan P (1995) Variation in the number, location and size of synaptic vesicles provides an anatomical basis for the nonuniform probability of release at hippocampal CA1 synapses. Neuropharmacology 34:1387-1395.

Heinemann C, von Rüden L, Chow RH, Neher E (1993) A two-step model of secretion control in neuroendocrine cells. Pflugers Arch 424:105-112.

Hempel CM, Hartman KH, Wang XJ, Turrigiano GG, Nelson SB (2000) Multiple forms of short-term plasticity at excitatory synapses in rat medial prefrontal cortex. J Neurophysiol 83:3031-3041.

Junge HJ, Rhee JS, Jahn O, Varoqueaux F, Spiess J, Waxham MN, Rosenmund C, Brose N (2004) Calmodulin and Munc13 form a Ca2+ sensor/effector complex that controls short-term synaptic plasticity. Cell 118:389-401.

Kalkstein JM, Magleby KL (2004) Augmentation increases vesicular release probability in the presence of masking depression at the frog neuromuscular junction. J Neurosci 24:11391-11403.

Klingauf J, Neher E (1997) Modeling buffered Ca2+ diffusion near the membrane: implications for secretion in neuroendocrine cells. Biophys J 72:674-690.

Klyachko VA, Stevens CF (2006a) Excitatory and feed-forward inhibitory hippocampal synapses work synergistically as an adaptive filter of natural spike trains. PLoS Biol 4:e207.

Klyachko VA, Stevens CF (2006b) Temperature-dependent shift of balance among the components of short-term plasticity in hippocampal synapses. J Neurosci 26:6945-6957.

Kusano K, Landau EM (1975) Depression and recovery of transmission at the squid giant synapse. J Physiol 245:13-32.

Losonczy A, Zhang L, Shigemoto R, Somogyi P, Nusser Z (2002) Cell type dependence and variability in the short-term plasticity of EPSCs in identified mouse hippocampal interneurones. J Physiol 542:193-210.

MacLeod KM, Horiuchi TK, Carr CE (2007) A role for short-term synaptic facilitation and depression in the processing of intensity information in the auditory brain stem. J Neurophysiol 97:2863-2874.

Magleby KL, Zengel JE (1976) Augmentation: a process that acts to increase transmitter release at the frog neuromuscular junction. J Physiol 257:449-470.

Magleby KL, Zengel JE (1982) A quantitative description of stimulationinduced changes in transmitter release at the frog neuromuscular junction. J Gen Physiol 80:613-638.

Mallart A, Martin AR (1967) An analysis of facilitation of transmitter release at the neuromuscular junction of the frog. J Physiol 193:679-694.

Markram H, Pikus D, Gupta A, Tsodyks M (1998) Potential for multiple mechanisms, phenomena, and algorithms for synaptic plasticity at single synapses. Neuropharmacology 37:489-500.

Matveev V, Zucker RS, Sherman A (2004) Facilitation through buffer saturation: constraints on endogenous buffering properties. Biophys J 86:2691-2709.

Murthy VN, Sejnowski TJ, Stevens CF (1997) Heterogeneous release properties of visualized individual hippocampal synapses. Neuron 18:599-612.

Neher E, Sakaba T (2008) Multiple roles of calcium ions in the regulation of neurotransmitter release. Neuron 59:861-872.

Oertner TG, Sabatini BL, Nimchinsky EA, Svoboda K (2002) Facilitation at single synapses probed with optical quantal analysis. Nat Neurosci 5:657-664.
O'Keefe J, Dostrovsky J (1971) The hippocampus as a spatial map. Preliminary evidence from unit activity in the freely-moving rat. Brain Res 34:171-175.

Pan B, Zucker RS (2009) A general model of synaptic transmission and short-term plasticity. Neuron 62:539-554.

Pouille F, Scanziani M (2001) Enforcement of temporal fidelity in pyramidal cells by somatic feed-forward inhibition. Science 293:1159-1163.

Rosenmund C, Sigler A, Augustin I, Reim K, Brose N, Rhee JS (2002) Differential control of vesicle priming and short-term plasticity by Munc13 isoforms. Neuron 33:411-424.

Sakaba T, Neher E (2001) Calmodulin mediates rapid recruitment of fastreleasing synaptic vesicles at a calyx-type synapse. Neuron 32:1119-1131.

Sara Y, Mozhayeva MG, Liu X, Kavalali ET (2002) Fast vesicle recycling supports neurotransmission during sustained stimulation at hippocampal synapses. J Neurosci 22:1608-1617.

Schikorski T, Stevens CF (1997) Quantitative ultrastructural analysis of hippocampal excitatory synapses. J Neurosci 17:5858-5867.

Schikorski T, Stevens CF (2001) Morphological correlates of functionally defined synaptic vesicle populations. Nat Neurosci 4:391-395.

Schneggenburger R, Sakaba T, Neher E (2002) Vesicle pools and short-term synaptic depression: lessons from a large synapse. Trends Neurosci 25:206-212.

Singer JH, Lassová L, Vardi N, Diamond JS (2004) Coordinated multivesicular release at a mammalian ribbon synapse. Nat Neurosci 7:826-833.

Sippy T, Cruz-Martín A, Jeromin A, Schweizer FE (2003) Acute changes in short-term plasticity at synapses with elevated levels of neuronal calcium sensor-1. Nat Neurosci 6:1031-1038.

Stevens CF, Wesseling JF (1998) Activity-dependent modulation of the rate at which synaptic vesicles become available to undergo exocytosis. Neuron 21:415-424.

Stevens CF, Wesseling JF (1999) Augmentation is a potentiation of the exocytotic process. Neuron 22:139-146.

Tong G, Jahr CE (1994) Multivesicular release from excitatory synapses of cultured hippocampal neurons. Neuron 12:51-59.

Tsodyks MV, Markram H (1997) The neural code between neocortical pyramidal neurons depends on neurotransmitter release probability. Proc Natl Acad Sci U S A 94:719-723.

Varela JA, Sen K, Gibson J, Fost J, Abbott LF, Nelson SB (1997) A quantitative description of short-term plasticity at excitatory synapses in layer $2 / 3$ of rat primary visual cortex. J Neurosci 17:7926-7940.

Wang XJ (1999) Fast burst firing and short-term synaptic plasticity: a model of neocortical chattering neurons. Neuroscience 89:347-362.

Weis S, Schneggenburger R, Neher E (1999) Properties of a model of $\mathrm{Ca}(++)$-dependent vesicle pool dynamics and short term synaptic depression. Biophys J 77:2418-2429.

Wesseling JF, Lo DC (2002) Limit on the role of activity in controlling the release-ready supply of synaptic vesicles. J Neurosci 22:9708-9720.

Wilson MA, McNaughton BL (1993) Dynamics of the hippocampal ensemble code for space. Science 261:1055-1058.

Zengel JE, Magleby KL (1977) Transmitter release during repetitive stimulation: selective changes produced by $\mathrm{Sr} 2+$ and $\mathrm{Ba} 2+$. Science 197:67-69.

Zengel JE, Magleby KL (1982) Augmentation and facilitation of transmitter release. A quantitative description at the frog neuromuscular junction. J Gen Physiol 80:583-611.

Zucker RS, Regehr WG (2002) Short-term synaptic plasticity. Annu Rev Physiol 64:355-405. 